\title{
Superposition rule and entanglement in diagonal and probability representations of density states $\ddagger$
}

\author{
Vladimir I. Man'ko, ${ }^{1}$ Giuseppe Marmo ${ }^{2}$ and E. C. George \\ Sudarshan $^{3}$ \\ ${ }^{1}$ P. N. Lebedev Physical Institute, Leninskii Prospect 53, Moscow 119991, \\ Russia \\ 2 Dipartimento di Scienze Fisiche, Università "Federico II" di Napoli \\ and Istituto Nazionale di Fisica Nucleare, Sezione di Napoli \\ Complesso Universitario di M. S. Angelo, Via Cintia, I-80126 Napoli, Italy \\ 3 Department of Physics, University of Texas, Austin, Texas 78712, USA \\ E-mail: mmanko@sci.lebedev.ru
}

\begin{abstract}
The quasidistributions corresponding to the diagonal representation of quantum states are discussed within the framework of operator-symbol construction. The tomographic-probability distribution describing the quantum state in the probability representation of quantum mechanics is reviewed. The connection of the diagonal and probability representations is discussed. The superposition rule is considered in terms of the density-operator symbols. The separability and entanglement properties of multipartite quantum systems are formulated as the properties of the density-operator symbols of the system states.

PACS numbers: 03.65.-w, 03.65.-Wj
\end{abstract}

\section{Introduction}

The pure quantum states are traditionally associated with the wave function [1] or a vector in the Hilbert space [2]. The mixed quantum states are described by the density matrix [3] or the density operator [4]. There exist several representations of quantum states in terms of the quasidistribution functions like the Wigner function [5] and the Husimi-Kano function [6, 7]. The diagonal representation of quantum states was suggested in [8] (see also [9]). It was studied and applied in [10, 11]. In this representation, a quantum state is represented in terms of weighted sum of coherentstate $|z\rangle$ projectors. The properties of all the quantum-state representations considered are associated with the properties of the density operator which is Hermitian, traceclass nonnegative operator. This means, in particular, that all the eigenvalues of the density operators must be nonnegative. In the quantum domain, the multipartite systems have a specific property connected with strong correlations of the quantum subsystems. This property provides the entanglement phenomenon [12].

In the diagonal representation of the density states, the weight function $\phi(z)$ is an analog of the probability-distribution function in the phase space. For some class of states, this function is identical to the probability-distribution function like in

$\ddagger$ based on the invited talk presented by one of us (V.I.M.) at the XV Central European Workshop on Quantum Optics (Belgrade, Serbia, 30 May - 3 June 2008). 
classical statistical mechanics. In [13], the tomographic-probability representation of quantum states, where the quantum state is associated with the so-called symplectic tomogram, was introduced. The tomogram is a fair probability distribution containing the same information on quantum state that the density operator does (or such its characteristics as the Wigner or Husimi-Kano functions). The aim of this work is to find the explicit formulae realizing the connection of the diagonal and tomographic probability representations. In [14], a review of the star-product-quantization schemes was given in a unified form. According to this scheme, the functions like the Wigner function, Husimi-Kano function and tomographic-probability-distribution function are considered as symbols of the density operators of a corresponding star-product scheme. The other goal of our work is to discuss in detail the diagonal representation within the framework of the star-product scheme along the lines of construction given in [14 and to find mutual relations between the tomographic-probability representation and the diagonal representation in this context. Using formulation of the superposition rule in terms of the density operator [15, 16, 17, we consider it within the framework of the density-state symbols. We focus on the superposition rule given in terms of tomograms and in terms of weight functions of the diagonal representation where explicit kernels of the corresponding star-products are employed to obtain the addition rules for the tomograms and weight functions. We discuss also the formulation of the separability and entanglement properties of composed system in the tomographic probability and diagonal representations.

The paper is organized as follows.

In Section 2, symplectic tomograms and the diagonal representation of quantum sates are reviewed. In Section 3, the superposition rule is considered. In Section 4, the diagonal representation and the star-product formalism are compared. In Section 5, the superposition rule for tomograms is presented. In Section 6, the entanglement in the tomographic and diagonal representations is studied. Conclusions are given in Section 7 .

\section{Symplectic tomogram and diagonal representation}

Below we review the approach where the quantum state associated with tomographic symbol (called symplectic tomogram) of the density operator (density state) $\hat{\rho}$ reads (see, for example, [17])

$$
w(X, \mu, \nu)=\operatorname{Tr} \hat{\rho} \delta(X \hat{1}-\mu \hat{q}-\nu \hat{p}) \quad(\hbar=1) .
$$

Here $X, \mu, \nu \in \mathrm{R}, \hat{q}$ and $\hat{p}$ are the position and momentum operators, respectively. For the pure state, tomogram is expressed in terms of the wave function 18

$$
w(X, \mu, \nu)=\frac{1}{2 \pi|\nu|}\left|\int \psi(y) \exp \left(\frac{i \mu}{2 \nu} y^{2}-\frac{i X y}{\nu}\right) d y\right|^{2} .
$$

The tomogram is nonnegative normalized probability distribution function of a random variable $X$, i.e.,

$$
w(X, \mu, \nu) \geq 0, \quad \int w(X, \mu, \nu) d X=1 .
$$

In the diagonal representation, the density state $\hat{\rho}$ reads 8

$$
\hat{\rho}=\int \phi(z)|z\rangle\langle z| d \operatorname{Re} z d \operatorname{Im} z,
$$


where $|z\rangle=\hat{D}(z)|0\rangle$ is the coherent state $\hat{a}|z\rangle=z|z\rangle$ and the displacement operator $\hat{D}(z)=\exp \left(z \hat{a}^{\dagger}-z^{*} \hat{a}\right)$ is called Weyl system. Here $\hat{a}=2^{-1 / 2}(\hat{q}+i \hat{p})$ is the boson annihilation operator and $z$ is a complex number. The probability distribution $w(X, \mu, \nu)$ is expressed in terms of the weight function $\phi(z)$ as follows:

$$
w(X, \mu, \nu)=\int \phi(z)\langle z|\delta(X \hat{1}-\mu \hat{q}-\nu \hat{p})| z\rangle d \operatorname{Re} z d \operatorname{Im} z .
$$

Using in (1) the Fourier decomposition of delta-function and taking the density state $\hat{\rho}$ in form (4), we obtain for tomogram

$$
w(X, \mu, \nu)=\frac{1}{2 \pi} \int \phi(z)\left\langle z\left|e^{i k(X-\mu \hat{q}-\nu \hat{p})}\right| z\right\rangle d \operatorname{Re} z d \operatorname{Im} z,
$$

where the diagonal matrix element of the operator in the integral can be considered as the Weyl-system matrix element, i.e.,

$$
\left\langle z\left|e^{-k(i \mu \hat{q}+i \nu \hat{p})}\right| z\right\rangle=\exp \left[z^{*} \alpha-\alpha^{*} z-\frac{|\alpha|^{2}}{2}\right],
$$

with

$$
\alpha=\frac{k}{\sqrt{2}}(\nu-i \mu) .
$$

Evaluating Gaussian integral (6), we arrive at

$$
\begin{aligned}
& w(X, \mu, \nu)=\frac{1}{\sqrt{\pi\left(\mu^{2}+\nu^{2}\right)}} \\
& \times \int \phi(z) \exp \left\{-\frac{\left[X-2^{-1 / 2}\left(z^{*}(\mu+i \nu)+z(\mu-i \nu)\right)\right]^{2}}{\mu^{2}+\nu^{2}}\right\} d \operatorname{Re} z d \operatorname{Im} z .
\end{aligned}
$$

The above formula provides the relation of the weight function of the diagonal representation of the density state and symplectic tomogram of the quantum state.

For example, the vacuum state $|0\rangle\langle 0|$ has the weight function

$$
\phi_{0}(z)=\delta(\operatorname{Re} z) \delta(\operatorname{Im} z) .
$$

Formula (9) provides tomogram of the ground state

$$
w_{0}(X, \mu, \nu)=\frac{1}{\sqrt{\pi\left(\mu^{2}+\nu^{2}\right)}} \exp \left(-\frac{X^{2}}{\mu^{2}+\nu^{2}}\right) .
$$

This expression can be obtained also by means of formula (2) with

$$
\psi_{o}(y)=\pi^{-1 / 4} \exp \left(-y^{2} / 2\right) \text {. }
$$

\section{Superposition rule for density operators}

For two orthogonal pure states $\left|\psi_{1}\right\rangle$ and $\left|\psi_{2}\right\rangle$, the superposition rule provides the state

$$
|\psi\rangle=\sqrt{p_{1}}\left|\psi_{1}\right\rangle+e^{i \phi} \sqrt{p_{2}}\left|\psi_{2}\right\rangle,
$$

which can be realized in the nature as a Schrödinger cat state. Here the positive numbers $p_{1}$ and $p_{2}$ satisfy the equality $p_{1}+p_{2}=1$ and the phase factor $e^{i \phi}$ determines 
the interference picture. The density states $\hat{\rho}_{1}=\left|\psi_{1}\right\rangle\left\langle\psi_{1}\right|$ and $\hat{\rho}_{2}=\left|\psi_{2}\right\rangle\left\langle\psi_{2}\right|$ provide the state $\hat{\rho}=|\psi\rangle\langle\psi|$, if one uses the nonlinear addition rule [15]

$$
\hat{\rho}=p_{1} \hat{\rho}_{1}+p_{2} \hat{\rho}_{2}+\sqrt{p_{1} p_{2}} \frac{\hat{\rho}_{1} \hat{P}_{0} \hat{\rho}_{2}+\hat{\rho}_{2} \hat{P}_{0} \hat{\rho}_{1}}{\sqrt{\operatorname{Tr}\left(\hat{\rho}_{1} \hat{P}_{0} \hat{\rho}_{2} \hat{P}_{0}\right)}},
$$

where the operator $\hat{P}_{0}$ is a projector $\left(\operatorname{Tr} \hat{P}_{0}=1\right)$ which corresponds to the phase term $e^{i \phi}$ in 11 .

The superposition rule can be formulated for any symbol of pure density states $\hat{\rho}_{1}, \hat{\rho}_{2}$ and $\hat{\rho}$.

\section{Diagonal representation and star-product formalism}

The diagonal representation of density operators can be considered within the framework of star-product scheme [14. Let us construct two families of operators, which are called dequantizer

$$
\hat{\mathcal{U}}(z)=\frac{1}{\pi^{2}} \int \exp \left(\frac{1}{2}|u|^{2}+z^{*} u-z u^{*}\right) \hat{D}(u) d \operatorname{Re} u d \operatorname{Im} u
$$

and quantizer

$$
\hat{\mathcal{D}}(z)=|z\rangle\langle z|,
$$

where $\hat{D}(u)$ is the Weyl system and $z=x+i y$ is a complex number. One can check that

$$
\operatorname{Tr} \widehat{\mathcal{U}}(z) \widehat{\mathcal{D}}\left(z^{\prime}\right)=\delta\left(x-x^{\prime}\right) \delta\left(y-y^{\prime}\right) .
$$

In view of this, one can construct the symbol of a density operator $\hat{\rho}$ in the diagonal representation

$\phi(z)=\operatorname{Tr} \widehat{\mathcal{U}}(z) \widehat{\rho}=\frac{1}{\pi^{2}} \int \exp \left(\frac{1}{2}|u|^{2}+z^{*} u-z u^{*}\right) \operatorname{Tr} \widehat{\rho} \widehat{D}(u) d \operatorname{Re} u d \operatorname{Im} u$

and the reconstruction formula for the density operator reads

$$
\widehat{\rho}=\int \phi(z)|z\rangle\langle z| d \operatorname{Re} z d \operatorname{Im} z .
$$

According to [19, 20], one can construct dual symbol of the operator $\widehat{\rho}$

$$
\phi^{(d)}(z)=\operatorname{Tr} \widehat{\rho}|z\rangle\langle z|=\langle z|\widehat{\rho}| z\rangle
$$

and dual reconstruction formula

$\widehat{\rho}=\int \phi^{(d)}(z) \widehat{\mathcal{U}}(z) d \operatorname{Re} z d \operatorname{Im} z$

$=\frac{1}{\pi^{2}} \int \phi^{(d)}(z) \exp \left(\frac{1}{2}|u|^{2}+z^{*} u-z u^{*}\right) \widehat{D}(u) d \operatorname{Re} u d \operatorname{Im} u d \operatorname{Re} z d \operatorname{Im} z$.

If in 16 the operator $\widehat{\rho}$ is replaced by some operator $\widehat{A}$, the corresponding symbol $\phi_{A}(z)$ provides the diagonal representation of the operator. The dual symbol 18 provides the Husimi-Kano function $Q(z)$. The reconstruction formula for the density state in terms of the Husimi-Kano function is just formula (19) with the replacement $\phi^{(d)}(z) \rightarrow Q(z)$. The duality relation of the diagonal representation of the density state $\widehat{\rho}$ and the Husimi-Kano function was discussed in [19, 21]. 
Using the connection of an operator symbol with its dual [14, one has the connection formula

$\phi(z)=\frac{1}{\pi^{3}} \int Q\left(z_{1}\right) \exp \left[|u|^{2}+\left(z^{*}-z_{1}^{*}\right) u-\left(z-z_{1}\right) u^{*}\right] d \operatorname{Re} u d \operatorname{Im} u$.

The inverse formula reads

$$
Q(z)=\int \phi(z) e^{-\left|z_{1}-z\right|^{2}} d \operatorname{Re} z_{1} d \operatorname{Im} z_{1} .
$$

According to the general formalism [14, the star-product of symbols related to the diagonal representation is determined by the kernel

$K\left(z_{1}, z_{2}, z\right)=\frac{1}{\pi^{2}} \operatorname{Tr}\left[\int\left|z_{1}\right\rangle\left\langle z_{1}|| z_{2}\right\rangle\left\langle z_{2}\right| \widehat{D}(u) \exp \left(\frac{1}{2}|u|^{2}+z^{*} u-z u^{*}\right) d \operatorname{Re} u d \operatorname{Im} u\right]$,

which is generalized function of the form

$$
\begin{aligned}
& K\left(z_{1}, z_{2}, z\right)=\frac{1}{\pi^{2}} \int \exp \left(-\left(x_{2}-x_{1}\right)^{2}-\left(y_{2}-y_{1}\right)^{2}+\left(x_{2}-x_{1}\right) a+\left(y_{2}-y_{1}\right) b\right. \\
& \left.-i\left(2 y+y_{1}+y_{2}\right) a+i\left(2 x+x_{1}+x_{2}\right) b\right) d a d b
\end{aligned}
$$

where

$$
z=x+i y, \quad z_{1}=x_{1}+i y_{1}, \quad z_{2}=x_{2}+i y_{2} .
$$

The star-product of symbols of arbitrary operators $\widehat{A}$ and $\widehat{B}$ in the diagonal representation reads

$\left(\phi_{A} * \phi_{B}\right)(z)=\int K\left(z_{1}, z_{2}, z\right) \phi_{A}\left(z_{1}\right) \phi_{B}\left(z_{2}\right) d x_{1} d y_{1} d x_{2} d y_{2}$.

For example, for the vacuum-state projector $\widehat{\rho}_{0}=|0\rangle\langle 0|$ with the weight function symbol $\phi_{0}(z)=\delta(z)$, one has

$\left(\phi_{0} * \phi_{0}\right)(z)=\int \delta\left(z_{1}\right) \delta\left(z_{2}\right) K\left(z_{1}, z_{2}, z\right) d x_{1} d y_{1} d x_{2} d y_{2}=\delta(z)$,

which is equal to $\phi_{0}(z)$ and corresponds to the pure-vacuum-state property $\widehat{\rho}_{0}^{2}=\widehat{\rho}_{0}$. Tomogram $w(X, \mu, \nu)$ of the density state $\hat{\rho}$ provides the following formula for the diagonal representation of the density operator

$\phi(z)=\frac{1}{2 \pi^{2}} \int w(X, \mu, \nu) \exp \left[i X+\frac{\mu^{2}+\nu^{2}}{4}+\frac{z(\nu+i \mu)}{\sqrt{2}}-\frac{z^{*}(\nu-i \mu)}{\sqrt{2}}\right] d X d \mu d \nu$.

For example, the vacuum-state tomogram

$$
w_{0}(X, \mu, 0)=\frac{1}{\pi\left(\mu^{2}+\nu^{2}\right)} \exp \left(-\frac{x^{2}}{\mu^{2}+\nu^{2}}\right)
$$

provides, by means of the above formula, the symbol of the state in the diagonal representation, i.e., $\delta(z)$. 


\section{Superposition rule for tomograms}

The superposition of two pure states with their symbols $\phi_{1}(z)$ and $\phi_{2}(z)$ is described by the function

$\phi(z)=p_{1} \phi_{1}(z)+p_{2} \phi_{2}(z)+\sqrt{p_{1} p_{2}} \frac{\left(\phi_{1} * \phi_{0} * \phi_{2}\right)(z)+\left(\phi_{2} * \phi_{0} * \phi_{1}\right)(z)}{\sqrt{\int\left(\phi_{1} * \phi_{0} * \phi_{2} * \phi_{0}\right)(z) d x d y}}$.

The star-product in (27) is determined by the kernel (23).

The result obtained can be repeated also for tomographic symbols of the density states. Thus, the addition rule of two tomographic probabilities of two pure states $\left|\psi_{1}\right\rangle$ and $\left|\psi_{2}\right\rangle$ reads

$w(X, \mu, \nu)=p_{1} w_{1}(X, \mu, \nu)+p_{2} w_{2}(X, \mu, \nu)$

$+\sqrt{p_{1} p_{2}} \frac{\left(w_{1} * w_{0} * w_{2}\right)(X, \mu, \nu)+\left(w_{2} * w_{0} * w_{1}\right)(X, \mu, \nu)}{\sqrt{\int \delta(\mu) \delta(\nu) d \mu d \nu d \mu^{\prime} d \nu^{\prime} \int e^{i X}\left(w_{1} * w_{0} * w_{2} * w_{0}\right)(X, \mu, \nu) d X}}$.

The kernel of tomographic star-product is given in [19, 20. The order of integration in the denominator term is essential to obtain the correct result. In (27) and (28), $\phi_{0}$ and $w_{0}$ are the corresponding symbols of projector $\widehat{P}_{0}$ which determines the relative phase of states $\left|\psi_{1}\right\rangle$ and $\left|\psi_{2}\right\rangle$ in their superposition.

\section{Entanglement in the diagonal and tomographic-probability representations}

Given bipartite system of a two-mode field.

The tomographic probability distribution is determined as follows:

$w\left(X_{1}, \mu_{1}, \nu_{1}, X_{2}, \mu_{2}, \nu_{2}\right)=\operatorname{Tr}\left[\widehat{\rho}(1,2) \delta\left(X_{1} \widehat{1}-\mu_{1} \widehat{q}_{1}-\nu_{1} \widehat{p}_{1}\right) \delta\left(X_{2} \widehat{1}-\mu_{2} \widehat{q}_{2}-\nu_{2} \widehat{p}_{2}\right)\right]$.

The density matrix in the diagonal representation is determined by the symbol of the density state $\widehat{\rho}(1,2)$

$\phi\left(z_{1}, z_{2}\right)=\frac{1}{\pi^{4}} \int \exp \left[\frac{1}{2}\left(\left|u_{1}\right|^{2}+\left|u_{2}\right|^{2}\right)+z_{1}^{*} u_{1}-z_{1} u_{1}^{*}+z_{2}^{*} u_{2}-z_{2} u_{2}^{*}\right]$

$\times \operatorname{Tr} \widehat{\rho}(1,2) \widehat{D}_{1}\left(u_{1}\right) \widehat{D}_{2}\left(u_{2}\right) d \operatorname{Re} u_{1} d \operatorname{Im} u_{1} d \operatorname{Re} u_{2} d \operatorname{Im} u_{2}$.

The state $\widehat{\rho}(1,2)$ is separable, if the density state can be written as a convex sum

$$
\widehat{\rho}(1,2)=\sum_{k} P_{k} \widehat{\rho}_{k}(1) \otimes \widehat{\rho}_{k}(2), \quad P_{k} \geq 0, \quad \sum_{k} P_{k}=1 .
$$

In view of linearity property of tomographic map, one has the definition of separability in terms of the state tomogram, i.e., the state is separable, if

$w\left(X_{1}, \mu_{1}, \nu_{1}, X_{2}, \mu_{2}, \nu_{2}\right)=\sum_{k} P_{k} w_{k}^{(1)}\left(X_{1}, \mu_{1}, \nu_{1}\right) w_{k}^{(2)}\left(X_{2}, \mu_{2}, \nu_{2}\right)$.

Tomogram is the joint probability-distribution function of two random variables $X_{1}, X_{2} \in \mathrm{R}$. Thus, the condition of the state separability is formulated as the above property 32 of the joint probability distribution.

If tomogram cannot be written as convex sum (32), the state is entangled. The separability condition can be reformulated, in view of the standard characteristic 
function for the tomographic probability distribution (32). In fact, if the characteristic function can be written as

$\chi\left(k_{1}, \mu_{1}, \nu_{1}, k_{2}, \mu_{2}, \nu_{2}\right)=\sum_{k} P_{k} \chi_{k}^{(1)}\left(k_{1}, \mu_{1}, \nu_{1}\right) \chi_{k}^{(2)}\left(k_{2}, \mu_{2}, \nu_{2}\right)$

the state is separable. Here $\chi_{k}^{(1)}\left(k_{1}, \mu_{1}, \nu_{1}\right)$ and $\chi_{k}^{(2)}\left(k_{2}, \mu_{2}, \nu_{2}\right)$ are the characteristic functions for tomographic probabilities $w_{k}^{(1)}\left(X_{1}, \mu_{1}, \nu_{1}\right)$ and $w_{k}^{(2)}\left(X_{2}, \mu_{2}, \nu_{2}\right)$, respectively.

An analogous definition of the separability and entanglement of the density state $\widehat{\rho}(1,2)$ can be formulated in the diagonal representation.

Thus the state is separable, if the function which is symbol of the density state in the diagonal representation can be written as

$$
\phi\left(z_{1}, z_{2}\right)=\sum_{k} P_{k} \phi_{k}^{(1)}\left(z_{1}\right) \phi_{k}^{(2)}\left(z_{2}\right) .
$$

Thus we formulated the problem of separability and entanglement in the diagonal representation of the density state $\widehat{\rho}(1,2)$. One can easily extend the definition of separable and entangled states to multipartite systems in both the tomographic and diagonal representations of density states.

\section{Conclusions}

To conclude, we resume the main results of this work.

We reviewed the diagonal and probability representations of quantum states using the standard star-product scheme. We found mutual relations of the weight function of the diagonal representation and the tomographic-probability distribution associated with the quantum state. We obtained the kernel of star-product of operator symbols in the diagonal representation. The duality relation between the diagonal representation of the weight function and the Husimi-Kano function was obtained in the explicit form. The superposition rule was formulated in both the diagonal representation and probability representation of the density states. The problem of separability and entanglement was formulated in both the diagonal and probability representations.

\section{Acknowledgments}

V.I.M. thanks the Russian Foundation for Basic Research for partial support under Projects Nos. 07-02-00598 and 08-02-90300 and the Organizers of the XV Central European Workshop on Quantum Optics (Belgrade, Serbia, 30 May - 3 June 2008) for kind hospitality.

\section{References}

[1] Schrödinger E 1926 Ann. Phys. (Liepzig) 79489

[2] Dirac P A M 1058 Principles of Quantum Mechanics 4th ed (London: Oxford University Press)

[3] Landau L D 1927 Z. Physik 45430

[4] von Neumann J 1932 Matematische Grundlagen der Quantenmechanyk (Berlin: Springer)

[5] Wigner E 1932 Phys. Rev. 40749

[6] Husimi K 1940 Proc. Phys. Math. Soc. Jpn 23264

[7] Kano Y 1956 J. Math. Phys. 61913

[8] Sudarshan E C G 1963 Phys. Rev. Lett. 10277

[9] Glauber R J 1963 Phys. Rev. Lett. 1084 
[10] Klauder J R and Sudarshan E C G 1968 Fundamentals of Quantum Optics (New York: Benjamin)

[11] Mehta C L and Sudarshan E C G 1965 Phys. Rev. 138 B274

[12] Schrödinger E 1935 Naturwissenchaften 23823

[13] Mancini S, Man'ko V I and Tombesi P 1996 Phys. Lett. A 2131

[14] Man'ko O V, Man'ko V I and Marmo G 2002 J. Phys. A: Math. Gen. 35699

[15] Man'ko V I, Marmo G, Sudarshan E C G and Zaccaria F 2002 J. Phys. A: Math. Gen. 357137

[16] Man'ko V I, Marmo G, Sudarshan E C G and Zaccaria F 2003 J. Russ. Laser Res. 24507

[17] Man'ko V I, Marmo G, Simoni A, Sudarshan E C G and Ventriglia F 2008 Rep. Math. Phys. 61337

[18] Man'ko V I and Mendes R V 1999 Phys. Lett. A 26353

[19] Man'ko V I, Marmo G and Vitale P 2005 Phys. Lett. A 3341

[20] Man'ko O V, Man'ko V I, Marmo G and Vitale P 2007 Phys. Lett. A 360522

[21] Klauder J R and Scagerstam B-S K 2007 J Phys. A: Math. Theor. 402093 